\title{
Combined positron emission tomography/computed tomography (PET/CT) for imaging of orbital tumours and tumours extending into the orbit
}

\author{
Annemarie Klingenstein, ${ }^{1}$ Gerd-Ullrich Mueller-Lisse, ${ }^{2}$ Alexander R Haug, ${ }^{3,4}$ \\ Aylin Garip-Kuebler, ${ }^{1}$ Christina V Miller, ${ }^{1}$ Christoph R Hintschich ${ }^{1}$
}

\begin{abstract}
Department of
Ophthalmology, Ludwig-

Maximilians-University,

Munich, Germany

2Department of Radiology,

Ludwig-Maximilians-University,

Munich, Germany

${ }^{3}$ Department of Nuclear

Medicine, Ludwig-Maximilians-

University, Munich, Germany

${ }^{4}$ Division of Nuclear Medicine,

Medical University Vienna,

Vienna, Austria
\end{abstract}

\section{Correspondence to}

Dr Annemarie Klingenstein,

Ludwig-Maximilians-University,

Department of Ophthalmology,

Klinikum der Universität

München, Campus Innenstadt,

Mathildenstraße 8, Munich D-

80336, Germany;

Annemarie.Klingenstein@med.

uni-muenchen.de

Received 15 October 2015 Revised 2 December 2015

Accepted 13 December 2015

Published Online First

14 January 2016

\section{CrossMark}

To cite: Klingenstein $A$, Mueller-Lisse G-U, Haug AR, et al. Br J Ophthalmol 2016;100:1403-1408

\begin{abstract}
Objective To assess clinical and radiological performance of combined positron emission tomography/ computed tomography (PET/CT) in patients with secondary and primary intraorbital tumours.
\end{abstract}

Methods 14 adults with secondary and 1 child with primary orbital masses underwent combined whole-body PET/CT. Radiopharmaceutical tracers applied were (18F)fluorodeoxyglucose, (18F)-fluoroethylcholine (FEC) and (68Ga)-DOTATATE. Histopathology and/or all conventional radiographic work-up and clinical course served as standard of reference. Descriptive statistics and Fisher's exact test were used for analysis.

Results PET/CT detected all orbital masses. All 15 patients had malignant disease. Local osseous infiltration was correctly identified in 11 patients. Lymph node metastases were present in two of eight patients (25\%) with haematogenous orbital metastases and in five of six patients (83\%) with infiltrative carcinoma ( $p=0.05)$. Further distant metastases were present in all eight patients suffering from orbital metastases, but only one patient with infiltrative carcinoma (17\%) presented with disseminated disease $(p=0.003)$. In one metastasis, PET/ CT excluded vital orbital tumour tissue after radiation therapy. Local recurrence was detected in another patient suffering from prostate cancer.

Conclusion PET/CT is a sensitive tool for the detection and localisation of orbital masses, enabling assessment of both morphology and cell metabolism. Detailed imaging of the head and neck region with a small field-of-view should be performed when suspecting lymphatic metastases. As metastatic disease to the orbit is associated with advanced disease, focus should be laid on wholebody imaging for staging of these patients. Different radiopharmaceutical tracers can be applied to distinguish the origin of orbital metastases.

\section{INTRODUCTION}

\section{Incidence and conventional imaging of orbital} tumours

Orbital tumours account for approximately $0.1 \%$ of all tumours and $18 \%$ of all orbital diseases. ${ }^{1}$ Contrast-enhanced CT and MRI can be considered as the standard imaging techniques to evaluate orbital tumours. ${ }^{2}$ Positron emission tomography (PET) with (18F)-fluorodeoxyglucose (FDG) is a well-established tool for staging and restaging of patients with cancer of the mouth, pharynx and larynx ${ }^{3}$ and can be applied for detecting residual tumour or recurrence and for the identification of lymph node involvement. ${ }^{4}$ Orbital metastases are rare in clinical practice ${ }^{5}$ and usually occur in disseminated disease. ${ }^{6}$ Nearly all solid malignancies have been reported to metastasise to the orbit, but the most frequent primary tumours in adults spreading to the orbit are breast cancer (42\%), lung cancer (11\%), cancer of unknown primary $(11 \%)$, prostate cancer $(8.3 \%)$, cutaneous melanoma (5.2\%), cancer of the gastrointestinal tract (4.4\%) and kidney cancer (3.2\%). ${ }^{1}$

\section{Combined PET/CT imaging}

In the past years, combined PET/CT imaging has emerged as a diagnostic modality and its importance has been proven in diagnosing and monitoring patients with different malignancies (eg, lymphoma, gastrointestinal tumours, non-small-cell lung cancer, cutaneous melanoma, breast cancer). ${ }^{7-10}$ PET/CT successfully combines data on metabolic activity obtained by PET with detailed morphological information gained from CT. Combined application helps to minimise the limitations of each modality by itself. ${ }^{711}$

Beyond (18F)-FDG, different other radiopharmaceutical tracers are available for PET/CT. (68Ga)-DOTATATE (68-Ga-1,4,7,10-tetraazacyclododecane-N,N',N",N'"-tetraacetic acid-d-Phe(1), $\operatorname{Tyr}(3)$-octreotate), a selective somatostatin receptor (SSTR) agonist, ${ }^{12}$ is applied for the detection of meningioma or neuroendocrine tumours. For imaging of other tumour entities that might metastasise to the orbit, specific radiopharmaceutical tracers such as, for example, (18F)-fluoroethylcholine ((18F)-FEC) or (68Ga)-PSMA (prostate-specific membrane antigen) in prostate cancer are applied regularly in clinical routine among others. ${ }^{13}$

\section{PET/CT for the detection of orbital tumours}

Since orbital tumours are rare disease entities, literature on the relevance of PET/CT for their detection to date is very limited. While PET/CT has already been considered as a new method for diagnosing, staging, and restaging patients with orbital lymphoma, ${ }^{14}$ the advantage in solid orbital tumours is regarded controversial. ${ }^{15}$ Our study group has reported on (68Ga)-DOTA-TATE PET/ $\mathrm{CT}$ as a useful tool in imaging orbital meningiomas previously. ${ }^{12}$ The objective of the current study was to evaluate the performance of the combined PET/ CT scanner in patients with primary and secondary solid orbital tumours.

\section{MATERIAL AND METHODS}

Patient selection

Selected patients were included retrospectively from our PET/CT database. We searched our PET/CT 
database via the keywords 'orbital tumors' and 'orbital pathology'. As we wanted to evaluate PET/CT performance of solid malignant tumours in this study, we then excluded all patients with orbital lymphoma. We included all other patients consecutively $(n=15)$.

This retrospective study has approval of the institutional review board of the Department of Ophthalmology of LudwigMaximilians-University of Munich/Germany. Written informed consent had been obtained from each adult patient; in the child, written informed consent had been obtained from parents, respectively.

\section{Indications for PET/CT scanning}

Since orbital masses are quite rare, patients included formed a heterogeneous group of diagnoses for whole-body PET/CT scanning: eight patients had metastatic cancer spreading to the orbit, six presented with tumours infiltrating into the orbit and one child presented with a primary orbital mass (for details see figure 1).

\section{PET/CT acquisition and image analysis}

PET/CT scanning was performed employing either a Philips Gemini System (Philips Medical Systems, Hamburg, Germany) or a Siemens Biograph TruePoint 64 (Siemens Medical Solutions, Erlangen, Germany) or a GE Discovery 690 (GE Healthcare, Munich, Germany). After fasting for $6 \mathrm{~h}, 20 \mathrm{mg}$ butylscopolamine (Buscopan; Boehringer Ingelheim, Ingelheim, Germany), $20 \mathrm{mg}$ furosemide and $250 \mathrm{MBq}$ (range 217$376 \mathrm{MBq}$ ) of the radiopharmaceutical tracer were applied intravenously in adults $((18 \mathrm{~F})-\mathrm{FDG}$ in $11,(68 \mathrm{Ga})$-DOTA-TATE in 1 and $(18 \mathrm{~F})-\mathrm{FEC}$ in 2 patients). In the child $110 \mathrm{MBq}$ of (18F)-FDG was administered. The imaging protocol included a low-dose CT scan for attenuation correction of PET imaging data acquired $60 \mathrm{~min}$ after intravenous administration of the PET tracer, a whole-body PET scan obtained with multiple bed positions, typically ranging from the top of the head to the upper third of the thigh and a diagnostic contrast-enhanced multidetector-row CT scan covering the range of the previous PET scan. Using the Philips Gemini PET/CT, low-dose CT scan settings were $20 \mathrm{~mA}, 140 \mathrm{kV}$ and $512 \times 512$ matrix; PET data were acquired with a field-of-view of $10 \mathrm{~cm}$ and $3 \mathrm{~min}$ per bed position and the diagnostic CT scan was performed with 100$190 \mathrm{~mA}$, depending on the scanned region, $120 \mathrm{kV}$, collimation $2 \times 5 \mathrm{~mm}$ and pitch 1.5 , in the venous phase, $75 \mathrm{~s}$ after intravenous injection of iodine-containing contrast agent (Ultravist 300; Schering, Berlin, Germany) at a rate of $2.5 \mathrm{~mL} / \mathrm{s}$. Similar protocols were applied with the other PET/CT scanners.

In one patient, due to known renal insufficiency, no contrast agent was employed. Also in the child, CT was performed without intravenous contrast agent. The reconstruction of the PET data was performed in three dimensional mode, with and without attenuation correction. CT data were evaluated alone, after multiplanar reformatting (axial, coronal, sagittal), and as combined PET/CT images as generated in with the Hermes system (Hermes Medical Solutions, London, UK). Evaluation of malignancy on CT was based on tumour morphology and patterns of contrast enhancement. For lymph nodes, CT criteria of malignancy included an axial diameter exceeding $10 \mathrm{~mm}$ and/or nodal clustering (three or more lymph nodes adjacent to each other), central necrosis and/or contrast enhancement. PET images were evaluated for regions of focally increased glucose uptake, typically with SUVmax exceeding a level of 5 .

\section{Validation of PET/CT findings}

Histology served as standard of reference in 10 patients. The remaining five patients had proven distant metastases from their underlying disease making orbital metastasis most probable. Whenever histopathology was not available, all previous and subsequent imaging studies (MRI, PET/CT) along with the clinical course and monitored treatment response served as standard of reference (for details, please see table 1).

\section{Statistical analysis}

Statistical analysis was performed using SPSS (V.23.0) for Windows (IBM; Armonk, New York, USA) employing Fisher's exact test and Microsoft Excel for Mac 2011.

\section{RESULTS}

\section{Demographic data}

Fifteen patients with orbital masses underwent whole-body PET/CT scanning.

Adult patients' median age was 70 years (range 46-79 years). We also included one boy aged 7 years at the time of PET/CT examination. Six patients had an orbital mass on the right eye, eight patients on the left eye and one patient presented with bilateral orbital infiltration. Eight patients were male and seven were female.

All orbital masses were detected by whole-body PET/CT (for patient details, see table 1). Osseous infiltration was noted in 11 orbital tumours (five metastases, five infiltrative carcinomas and one rhabdomyosarcoma).

\section{$\mathrm{PET} / \mathrm{CT}$ in rhabdomyosarcoma $(\mathrm{n}=1)$}

Patient number 9 suffered from histology-proven rhabdomyosarcoma which infiltrated the roof and the apex of the orbit. Clinical ophthalmological examination revealed papilloedema and exophthalmos. PET/CT correctly ruled out systemic metastasis.

\section{PET/CT in secondary orbital tumours $(n=14)$}

Whole-body PET/CT correctly assessed six patients with continuous tumour spread to the orbit and eight orbital metastases. Histology-proven primary tumours of these patients included breast cancer $(n=3)$, prostate cancer $(n=2)$, invasive follicular thyroid carcinoma $(n=1)$, cutaneous melanoma $(n=1)$ and neuroendocrine carcinoma of the small intestine $(n=1)$. While (18F)-FDG accumulation indicates an elevated glucose metabolism in general, (18F)-FEC and (68Ga)-DOTATATE were specifically employed for suspected metastasis of prostate cancer and neuroendocrine carcinoma, respectively. Further metastatic sites in patients with orbital metastases included osseous $(n=8)$, hepatic $(n=4)$, pulmonary $(n=2)$ as well as one cutaneous, muscular and endometrium metastasis, respectively.

Lymph node metastases were present in two of eight patients with haematogenous tumour spread $(25 \%)$ and in five of six patients with continuous tumour spread (83\%). Lymph node involvement was more frequent in patients with local carcinomas extending into the orbit (Fisher's exact test, $\mathrm{p}=0.05$ ). Further organ involvement was present in all eight patients suffering from orbital metastases, whereas only one patient with infiltrative carcinoma had further distant metastases (17\%). This was highly significant (Fisher's exact test, $p=0.003$ ). These results are summarised in table 2 .

In metastatic patients, seven orbital metastases expressed an elevated metabolism indicating vital tumour tissue and consequently leading to treatment decision. In one patient, PET/CT could exclude vital orbital tumour tissue after radiation therapy (patient number 13, figure 2), yet further distant lymph node, pulmonary as well as osseous metastases were present. The patient then underwent chemotherapy. 


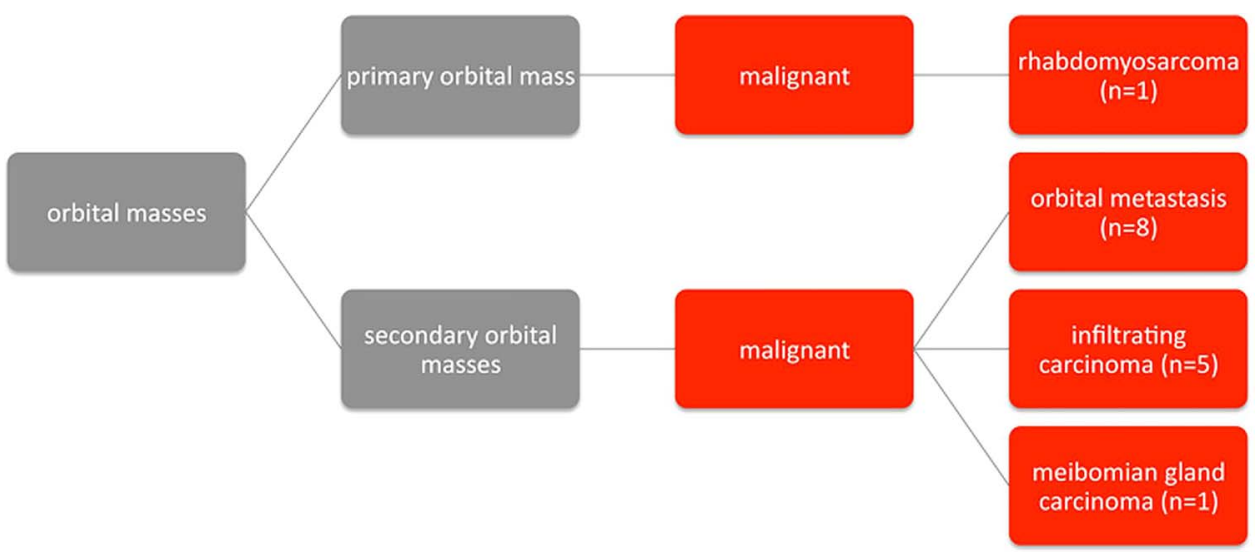

Figure 1 Classification of final diagnoses of orbital lesions included $(n=15)$.

In patient 1 suffering from prostate cancer, previously unknown local recurrence was detected by (18F)-FEC PET/CT (figure 3).

Thus, PET/CT had influence on treatment decision in all patients.

\section{DISCUSSION}

Combined morphological and metabolic data in PET/CT improve diagnostic accuracy. This plays an important role in the selection of adequate therapy. Higher patient convenience, saving of time and greater diagnostic confidence are important advantages of combined imaging. ${ }^{16}$

\section{$\mathrm{PET} / \mathrm{CT}$ in the child presenting with an orbital mass}

In spite of the radiation exposure, PET/CT with reduced dose $(110 \mathrm{MBq})$ was applied in one case of a primary orbital tumour.
The child was symptomatic and presented with apparent exophthalmos and papilloedema implied neoplastic growth. For paediatric rhabdomyosarcoma, PET/CT has already been proven superior to conventional radiological evaluation in the detection of distant metastases ${ }^{17}$ and lymph node metastases ${ }^{18}$ and was superior in detecting objective response after chemotherapy compared with conventional imaging methods (92\% vs $84 \%) .{ }^{18}$ Whole-body (18F)-FDG PET/CT may be useful in detecting metastases of rhabdomyosarcoma that are not clinically evident and upstaging in comparison with MRI staging (lymph node and osseous metastases) has been reported previously. ${ }^{19}$ PET/CT has thus been proven a meaningful tool in the staging of carcinoma and rhabdomyosarcoma. $^{20}$ In the child with rhabdomyosarcoma in this study, PET/ CT was successfully employed for staging (exclusion of further distant metastasis) and restaging after chemotherapy.

Table 1 Overview of patients $(n=15)$ with primary and secondary orbital masses who underwent combined PET/CT

\begin{tabular}{|c|c|c|c|c|c|c|c|c|c|}
\hline $\begin{array}{l}\text { Pt. ID } \\
(n=15)\end{array}$ & $\begin{array}{l}\text { Gender } \\
(m: f=8: 7)\end{array}$ & $\begin{array}{l}\text { Age at } \\
\text { PET/CT } \\
\text { (y) }\end{array}$ & Therapy & Eye & $\begin{array}{l}\text { Tumour localisation } \\
\text { within the orbit }\end{array}$ & Final diagnosis & PET-Tracer & Validation & $\begin{array}{l}\text { Ocular } \\
\text { symptoms }\end{array}$ \\
\hline 1 & M & 68 & CyberKnife & OS & Superiorly & Metastasis (prostate cancer) & (18F)-FEC & MRI & BCVA $\downarrow$ \\
\hline 2 & $\mathrm{M}$ & 71 & $\begin{array}{l}\text { CyberKnife, hormone } \\
\text { therapy }\end{array}$ & OD & Temporally & Metastasis (prostate cancer) & (18F)-FEC & MRI & None \\
\hline 3 & $\mathrm{~F}$ & 62 & $\begin{array}{l}\text { CyberKnife, } \\
\text { chemotherapy }\end{array}$ & OS & Inferiorly & Metastasis (breast cancer) & (18F)-FDG & MRI, PET/CT & None \\
\hline 4 & $\mathrm{~F}$ & 46 & $\begin{array}{l}\text { Surgical resection, } \\
\text { radiation therapy }\end{array}$ & OD & Medially & Neuroendocrine carcinoma & (18F)-FDG & Histopathology & VF defects \\
\hline 5 & $\mathrm{~F}$ & 60 & $\begin{array}{l}\text { Surgical resection, } \\
\text { radiation therapy }\end{array}$ & OS & Superiorly & Metastasis (thyroid cancer) & (18F)-FDG & Histopathology & Double vision \\
\hline 6 & $\mathrm{M}$ & 72 & $\begin{array}{l}\text { Radiation therapy, } \\
\text { DOTA therapy }\end{array}$ & OS & Temporally & $\begin{array}{l}\text { Metastasis (neuroendocrine } \\
\text { carcinoma) }\end{array}$ & (68Ga)-DOTATATE & MRI & None \\
\hline 7 & $\mathrm{~F}$ & 58 & $\begin{array}{l}\text { Radiation therapy, } \\
\text { hormone therapy }\end{array}$ & OD & Medially & Metastasis (breast cancer) & (18F)-FDG & MRI & Enophthalmos \\
\hline 8 & M & 71 & Surgical resection & OD & Medially & Squamous cell carcinoma & (18F)-FDG & Histopathology & Skin alteration \\
\hline 9 & $\mathrm{M}$ & 7 & Chemotherapy & OS & Superiorly, orbital apex & Rhabdomyosarcoma & (18F)-FDG & Histopathology & Exophthalmos \\
\hline 10 & $\mathrm{M}$ & 70 & Radiation therapy & OD & Medially & Nasopharyngeal carcinoma & (18F)-FDG & Histopathology & BCVA $\downarrow$ \\
\hline 11 & $\mathrm{~F}$ & 79 & Surgical resection & OS & Superiorly, medially & Meibomian gland carcinoma & (18F)-FDG & Histopathology & Inflammation \\
\hline 12 & $\mathrm{M}$ & 76 & Surgical resection & OD & Medially, lacrimal duct & Squamous cell carcinoma & (18F)-FDG & Histopathology & Skin alteration \\
\hline 13 & $\mathrm{~F}$ & 76 & $\begin{array}{l}\text { Chemotherapy, } \\
\text { Radiotherapy }\end{array}$ & OS & Superiorly & Metastasis (breast cancer) & (18F)-FDG & Histopathology & Ptosis \\
\hline 14 & $\mathrm{M}$ & 68 & Chemotherapy & $\begin{array}{l}\text { OD } \\
\text { OS }\end{array}$ & Medially & Neuroendocrine carcinoma & (18F)-FDG & Histopathology & Double vision \\
\hline 15 & $\mathrm{~F}$ & 76 & $\begin{array}{l}\text { Radiation therapy, } \\
\text { immunotherapy }\end{array}$ & OS & Orbital apex & $\begin{array}{l}\text { Metastasis (cutaneous } \\
\text { melanoma) }\end{array}$ & (18F)-FDG & Histopathology & BCVA $\downarrow$ \\
\hline
\end{tabular}


Table 2 Tumour spread in adults with orbital metastases vs tumours extending into the orbit

\begin{tabular}{llll}
\hline & $\begin{array}{l}\text { Orbital } \\
\text { metastases } \\
(\mathbf{n}=8)\end{array}$ & $\begin{array}{l}\text { Tumours } \\
\text { extending into } \\
\text { the orbit } \\
(\mathbf{n}=6)\end{array}$ & $\begin{array}{l}\mathbf{p} \text { Value } \\
\text { (Fisher's } \\
\text { exact test) }\end{array}$ \\
\hline Bony infiltration & $5(63 \%)$ & $5(83 \%)$ & n.s. \\
Lymph node metastases & $2(25 \%)$ & $5(83 \%)$ & 0.05 \\
Further distant metastases & $8(100 \%)$ & $1(17 \%)$ & 0.003 \\
\hline n.s., not significant. & & &
\end{tabular}

\section{$\mathrm{PET} / \mathrm{CT}$ for detection of lymph node metastases} in orbital tumours

We employed PET/CT for diagnosing metastasis to the orbit and for detecting the extent of lymph node involvement from invasive malignancies. This has been widely evaluated for the head and neck region in cervical carcinoma of unknown primary. ${ }^{21}$ PET/CT has the potential to improve the management of patients with carcinoma in the sinus/nasal area and orbit. ${ }^{2}$ Variable diagnostic accuracy has been shown for (18F)-FDG PET in the detection of different histological tumour entities. ${ }^{22}$

Lymph node staging has extensive consequences for the patients ranging from no adjuvant therapy to very radical therapy like neck dissection, brachytherapy or chemotherapy in advanced tumours. Advantages of lymph node evaluation via PET/CT versus sentinel lymph node biopsy are of interest for the tumours included in our study that extended into the orbit. PET/CT is less invasive than biopsy enabling whole-body evaluation within the same examination. Furthermore, indication for sentinel lymph node biopsy in conjunctival and eyelid tumours is limited to specific tumour stages, according to a recent review, and multi-institutional controlled trials are warranted..$^{23}$ Data on sentinel lymph node biopsy on tumours extending into the orbit are even more limited. A comparative study on the sensitivity and specificity of sentinel lymph node biopsy and lymph node staging via PET/CT would be of great value. Additional lymph

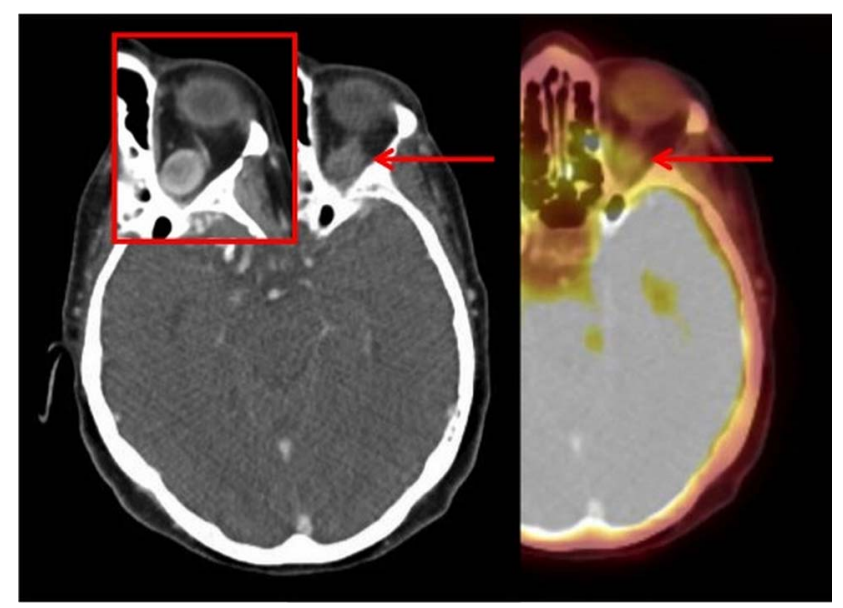

Figure 2 Intraorbital breast cancer metastasis invading the levator complex demonstrating low fluorodeoxyglucose uptake after radiation therapy (patient 13). The CT scan on the left shows the orbital metastasis before (within the red frame) and after radiotherapy. The scan on the right is taken from the same examination after therapy but is showing combined positron emission tomography/CT imaging. node metastases may modify treatment decision (neck dissection or delineation of the radiation treatment field).

\section{Field-of-view in PET/CT}

In clinical practice in both nuclear medicine and radiology, increasing spatial resolution of images is achieved by decreasing the field-of-view, because the whole reconstruction matrix is used for a smaller anatomical region. Consequently, this reduces pixel size within the images. The field-of-view reflects the maximum diameter of the reconstructed digital image. Its value can be selected by the radiologist depending on the dimensions of the anatomical region of interest. The choice of a small field-of-view allows increased spatial resolution within the image, because the whole reconstruction matrix is used for a smaller region. A large field-of-view is usually selected for whole-body-PET/CT imaging to fully include even the widest axial dimensions of the human body, that is, the shoulders and the hips, in search of distant metastasis. In this study, as orbital metastases were associated with further distant metastases in all cases, a large field-of-view in PET/CT was reasonable in order not to overlook any distant metastasis. However, detailed imaging of the head and neck region was of utmost importance in lesions extending into the orbit. These infiltrative tumours were more likely to develop lymph node metastases rather than distant metastases. Therefore, additional image reconstructions with a small field-of-view were chosen to cover the head and neck regions in these patients with high spatial resolution. Whenever imaging raw data are kept available, the field-of-view can be changed in retrospect.

\section{$\mathrm{PET} / \mathrm{CT}$ in orbital metastases}

The actual incidence of metastases to the eye and orbit is difficult to quantify, as patients may remain asymptomatic and succumb to their primary cancer before clinical detection of the ocular metastasis. Yet, with improvements in systemic therapy and oncological management, patients suffering from metastatic cancer survive longer and, therefore, orbital metastases may manifest more frequently. ${ }^{24}$

Of course, clinical examination remains the first important method for evaluation of orbital symptoms. However, for biopsy planning, CT is necessary for assessment of bony invasion or if the tumour is localised in the orbital apex, making ultrasound examination difficult.

Whole-body PET/CT imaging can be employed for detecting primary site in metastatic disease and defining the total extent of disseminated disease. PET/CT can indicate vital tumour metabolism, and selectively binding radiopharmaceutical tracers, which were applied for diagnostics of prostate cancer or neuroendocrine tumour metastases in this study, can determine the origin of tumour cells. PET may overcome CT limitations by its ability to visualise vital tumour tissue by increased FDG uptake and therefore identify residual cancer or recurrence. ${ }^{2}$ In this study, recurrence of prostate cancer was detected on (18F)-FEC PET/CT in one patient, thus modifying treatment. In a large retrospective study evaluating different non-typical sites of metastasis of neuroendocrine tumours, the most common locations were cardiac and breast metastases followed by retroorbital metastases. ${ }^{25}$ In cases with known neuroendocrine tumours, (68Ga)-DOTATATE PET/CT can delineate the orbital mass in question and can determine tumour biology in terms of SSRT expression. In the present study, a previously unknown orbital metastasis could be diagnosed correctly as such with (68Ga)-DOTATATE PET/CT.

In PET/CT, detection of distant metastases may be performed within the same whole-body scan. This has been reported in 
Figure 3 Temporal orbital metastasis as shown on CT and positron emission tomography (PET) (above, patient 1). The CT scan on the left is showing bony infiltration by the orbital mass. The PET scan on the right is demonstrating obviously elevated tracer uptake of the lesion in question. The CT scan on the left (below) is showing a previously unknown local recurrence of prostate cancer and the (18F)-fluoroethylcholine PET image on the right demonstrates its elevated tumour metabolism.
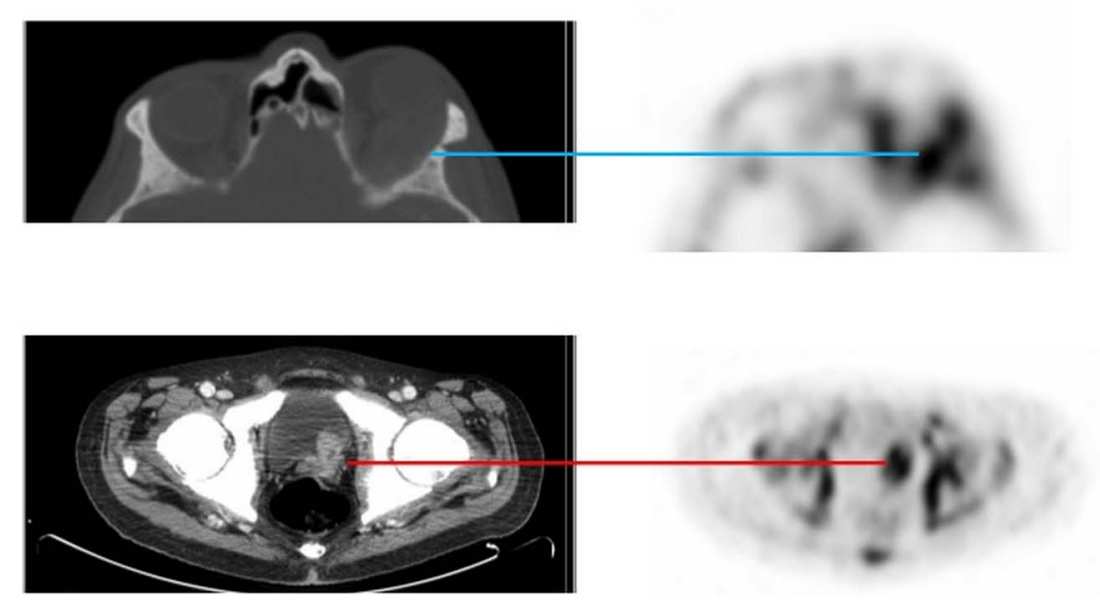

$27 \%$ of patients by Wild et $a l^{2}$ for carcinomas of the sinus/nasal area and orbit. $^{2}$ The group reported superior performance of PET/CT for restaging locoregional carcinoma of the sinus, nasal area and orbit, but not for initial staging.

We found additional systemic metastases present in all eight patients who presented with orbital metastases (100\%) within this study. Orbital metastases usually spread hematogenously. ${ }^{6}$ Disseminated disease was significantly more frequent in patients with haematogenous orbital metastases than in patients with infiltrative tumour growth $(p=0.003)$. We therefore conclude that whole-body PET/CT including imaging of the head and orbit should be employed in patients with systemic metastases presenting with recent orbital symptoms. Additionally, PET/CT should be performed in patients with tumour history without known metastasis and orbital symptoms as these symptoms can be the first manifestation of diffuse systemic metastases. PET/CT seems most appropriate for advanced cancers of the orbit, eyelid and eye with high risk of distant metastasis. ${ }^{15}$

\section{CONCLUSION}

By employing PET/CT, both tumour morphology and cell metabolism can be assessed in one single examination, thereby helping in overcoming the limitations of each modality used separately. As orbital metastases did not present as solitary findings in the present study, whole-body staging is of great value for the evaluation of metastatic spread and specific radiopharmaceutical tracers can be employed for this purpose. For infiltrating tumours associated with lymph node metastases, PET/ CT has proven valuable for the evaluation of lymph node involvement. Additionally, PET/CT can be of importance in particular settings of paediatric ocular diagnostics.

Correction notice This article has been corrected since it was published Online First. The first two column headings in table 1 have been corrected from Pt. ID $(n=16)$ and Gender (m:f=8:6) to Pt. ID $(n=15)$ and Gender (m:f=8:7), respectively.

Contributors All authors (AK, G-UM-L, ARH, AG-K, CVM and CRH) made substantial contributions to the conception and design of the manuscript and the acquisition, analysis and interpretation of data for the work and drafting the work and critical revision for important intellectual content. They have approved the final version to be published and have agreed to be accountable for all aspects of the work in ensuring that questions related to the accuracy or integrity of any part of the work are appropriately investigated and resolved.

Competing interests None declared.

Ethics approval Institutional review board of Ophthalmology.

Provenance and peer review Not commissioned; externally peer reviewed.

\section{REFERENCES}

1 Goldberg RA, Rootman J, Cline RA. Tumors metastatic to the orbit: a changing picture. Surv Ophthalmol 1990;35:1-24.

2 Wild D, Eyrich GK, Ciernik IF, et al. In-line (18)F-fluorodeoxyglucose positron emission tomography with computed tomography (PET/CT) in patients with carcinoma of the sinus/nasal area and orbit. J Craniomaxillocac Surg 2006;34(Suppl 1):9-16.

3 McGuirt WF, Greven K, Williams D III, et al. PET scanning in head and neck oncology: a review. Head Neck 1998;20:208-15.

4 Lonneux M, Lawson G, Ide C, et al. Positron emission tomography with fluorodeoxyglucose for suspected head and neck tumor recurrence in the symptomatic patient. Laryngoscope 2000;110:1493-7.

5 Shields JA, Shields CL, Brotman HK, et al. Cancer metastatic to the orbit: the 2000 Robert M. Curts Lecture. Ophthal Plast Reconstr Surg 2001; 17:346-54.

6 Manohar K, Mittal BR, Bhattacharya A, et al. Orbital Metastases as Presenting Sign of Lung Carcinoma: Detection of Primary Malignancy and Disease Burden by F-18 FDG PET/CT. Nucl Med Mol Imaging 2012;46:73-5.

7 Antoch G, Saoudi N, Kuehl H, et al. Accuracy of whole-body dual-modality fluorine-18-2-fluoro-2-deoxy-D-glucose positron emission tomography and computed tomography (FDG-PET/CT) for tumor staging in solid tumors: comparison with CT and PET. J Clin Oncol 2004;22(Suppl 21): 4357-68.

8 Manning K, Tepfer B, Goldklang G, et al. Clinical practice guidelines for the utilization of positron emission tomography/ computed tomography imaging in selected oncologic applications: suggestions from a provider group. Mol Imaging Biol 2007;9(Suppl 6):324-32.

9 Schöder H, Larson SM, Yeung HW. PET/computed tomography in oncology: integration into clinical management of lymphoma, melanoma and gastrointestinal malignancies. J Nucl Med 2004;45(Suppl 1):72-81.

10 Haug AR, Schmidt GP, Klingenstein A, et al. F-18-Fluoro-2-Deoxyglucose Positron Emission Tomography/Computed Tomography in the Follow-up of Breast Cancer With Elevated Levels of Tumor Markers. J Comput Assist Tomogr 2007;31:629-34.

11 Klingenstein A, Haug AR, Nentwich MM, et al. Whole-body F-18-fluoro-2-deoxyglucose positron emission tomography/computed tomography imaging in the follow-up of metastatic uveal melanoma. Melanoma Res 2010;20 (Suppl 6):511-16.

12 Klingenstein A, Haug AR, Miller C, et al. Ga-68-DOTA-TATE PET/CT for discrimination of tumors of the optic pathway. Orbit 2015;34(Suppl 1): $16-22$.

13 Fernandes F, da Silva D, Rodrigues L. SU-E-I-82: PET Radiopharmaceuticals for Prostate Cancer Imaging: A Review. Med Phys 2015;42(Suppl 6): 3261.

14 Roe RH, Finger PT, Kurli M, et al. Whole-body positron emission tomography/ computed tomography imaging and staging of orbital lymphoma. Ophthalmology 2006;113(Suppl 10):1854-8.

15 Hui KH, Pfeiffer ML, Esmaeli B. Value of positron emission tomography/computed tomography in diagnosis and staging of primary ocular and orbital tumors. Saudi J Ophthalmol 2012;26(Suppl 4):365-71.

16 Townsend DW, Beyer T, Blodgett TM. PET/CT scanners: a hardware approach to image fusion. Semin Nucl Med 2003;33:193-204.

17 Tateishi U, Hosono A, Makimoto A, et al. Comparative study of FDG PET/CT and conventional imaging in the staging of rhabdomyosarcoma. Ann NuCl Med 2009;23 (Suppl 2):155-61. 


\section{Clinical science}

18 Eugene T, Corradini N, Carlier T, et al. ${ }^{18} \mathrm{~F}$-FDG-PET/CT in initial staging and assessment of early response to chemotherapy of pediatric rhabdomyosarcomas. Nucl Med Commun 2012;33(Suppl 10):1089-95.

19 Ozcan Kara P, Kara Gedik G, Sari O. Detection of bone metastases in initial staging of orbital embryonal rhabdomyosarcoma by fluorodeoxyglucose positron emission tomography/computed tomography. Mol Imaging Radionucl Ther 2011;20 (Suppl 1):34-5.

20 Hassan WM, Alfaar AS, Bakry MS, et al. Orbital tumors in USA: difference in survival patterns. Cancer Epidemiol 2014;38:515-22.

21 Deron $\mathrm{PB}$, Bonte $\mathrm{KM}$, Vermeersch $\mathrm{HF}$, et al. Lymph node metastasis of squamous cell carcinoma from an unknown primary in the upper and middle neck: impact of (18) F-fluorodeoxyglucose positron emission tomography/computed tomography. Cancer Biother Radiopharm 2011;26:331-4.
22 Bui CD, Ching AS, Carlos RC, et al. Diagnostic accuracy of 2-[fluorine-18] fluoro-2-deoxy-D-glucose positron emission tomography imaging in nonsquamous tumors of the head and neck. Invest Radiol 2003; 38:593-601.

23 Pfeiffer ML, Savar A, Esmaeli B. Sentinel Lymph Node Biopsy for Eyelid and Conjunctival Tumors: What Have We Learned in the Past Decade? Ophthal Plast Reconstr Surg 2013;29:57-62.

24 Rudoler SB, Shields CL, Shields JA. Radiation therapy of uveal and orbital metastases. In: Sagerman RH, Alberti WE, eds. Radiotherapy of Intraocular and Orbital Tumors. Berlin Heidelberg New York: Springer, 2003:87-93.

25 Carreras C, Kulkarni HR, Baum RP. Rare metastases detected by [68Ga]-somatostatin receptor PET/CT in patients with neuroendocrine tumors. Recent Results Cancer Res 2013;194:379-84. 